Cite this: Food Funct., 2021, 12, 9018
(D) Check for updates

\section{Chicory inulin enhances fermentation of 2'-fucosyllactose by infant fecal microbiota and differentially influences immature dendritic cell and T-cell cytokine responses under normal and Th2-polarizing conditions $\uparrow$}

\author{
Renate Akkerman, (DD * * Madelon J. Logtenberg, ${ }^{*} t^{\mathrm{b}}$ Martin Beukema, (D) a \\ Bart J. de Haan, ${ }^{a}$ Marijke M. Faas, ${ }^{a}$ Erwin G. Zoetendal, ${ }^{C}$ Henk A. Schols ${ }^{b}$ and \\ Paul de Vos ${ }^{a}$
}

\begin{abstract}
Scope: Non-digestible carbohydrates (NDCs) such as native chicory inulin and 2'-fucosyllactose (2'-FL) are added to infant formula to mimic some of the human milk oligosaccharide (HMO) functions. It is unknown whether combining inulin and 2'-FL influences their fermentation kinetics and whether the immune-modulatory effects of these NDCs are different under normal and inflammatory-prone Th2polarizing conditions. Methods and results: We investigated the in vitro fermentation of 2'-FL and native chicory inulin, fermented individually and combined, using fecal inocula of 8 -week-old infants. Native inulin was fermented in a size-dependent fashion and expedited the fermentation of 2'-FL. Fermentation of both native inulin and 2'FL increased the relative abundance of Bifidobacterium, which coincided with the production of acetate and lactate. The fermentation digesta of all fermentations differentially influenced both dendritic cell and T-cell cytokine responses under normal culture conditions or in presence of the Th2-polarizing cytokines IL-33 and TSLP, with the most pronounced effect for IL-1 $\beta$ in the presence of TSLP. Conclusions: Our findings show that native inulin can expedite the fermentation of 2'FL by infant fecal microbiota and that these NDC fermentation digesta have different effects under normal and Th2-polarizing conditions, indicating that infants with different immune backgrounds might benefit from tailored NDC formulations.
\end{abstract}

Received 23rd March 2021, Accepted 2nd August 2021 DOI: $10.1039 / \mathrm{d} 1 \mathrm{fo} 00893 \mathrm{e}$ rsc.li/food-function

\section{Introduction}

The non-digestible carbohydrates (NDC) inulin-type fructans and the human milk oligosaccharide (HMO) 2'-fucosyllactose (2'-FL) are added to infant formulas to mimic some of the effects of HMOs in human milk (HM). Although having many different beneficial effects, these NDCs are mainly added to infant formula to mimic HMO-induced prebiotic and immune stimulating properties in new-born infants. ${ }^{1,2}$ Inulin-type fruc-

\footnotetext{
ammunoendocrinology, Division of Medical Biology, Department of Pathology and Medical Biology, University of Groningen and University Medical Centre Groningen, Groningen, The Netherlands. E-mail: r.akkerman@umcg.nl

${ }^{b}$ Laboratory of Food Chemistry, Wageningen University \& Research, Wageningen, The Netherlands. E-mail: madelon.logtenberg@wur.nl

${ }^{c}$ Laboratory of Microbiology, Wageningen University \& Research, Wageningen, The Netherlands

$\dagger$ Electronic supplementary information (ESI) available. See DOI: 10.1039/ d1fo00893e

\$Authors contributed equally.
}

tans contain a range of different short- and long-chain fructan molecules, ${ }^{3}$ and are added alone or in combination with galacto-oligosaccharides (GOS) to infant formula, ${ }^{4}$ while the HMO 2'-FL is more recently added to infant formulas as a single molecule. ${ }^{5}$ To date, there are only a few combinations of different NDC and/or HMOs being used in infant formulas, while there are many other potentially interesting combinations possible. The application of new combinations of NDCs and HMOs can be of interest to tailor infant formulas for specific groups of infants as it was shown that NDCs and HMOs all have their own unique structure- and infant-agedependent immune effects. ${ }^{6-8}$

Inulin-type fructans can be found in many natural sources including beet roots, leeks and banana, but is mainly extracted from chicory roots. They are composed of linear chains of (21)-linked fructose monomers and either have a glucose or a fructose moiety at the reducing end, ${ }^{3}$ with chains with a varying degree of polymerization (DP). Native chicory inulin is a commercially available fructan mixture which predominantly 
contains molecules with a DP range from 2 to 60 and with a glucose moiety at the reducing end. There are studies available showing that inulin-type fructans can directly modulate immune responses in a chain length dependent manner.,10 Next to direct immune effects, fermentation of inulin-type fructans can also indirectly influence immune responses, as fermentation of inulin-type fructans, particularly by Bifidobacterium species, results in the formation of short chain fatty acids (SCFAs) and other bacterial metabolites. ${ }^{11,12}$ In a previous study, we showed that the capacity of the infant's microbiota to ferment native inulin is age-dependent and that the fermentation of native inulin could attenuate pro-inflammatory responses in DCs. ${ }^{6}$

Next to native inulin, the HMO 2'-FL is now added to infant formulas. ${ }^{5}$ Since a few years it is possible to produce 2 -FL as a fermentation product of genetically engineered microorganisms such as E. coli and yeast, ${ }^{13,14}$ which makes it possible to produce it in a cost-effective way which allows broad application in infant formulas. ${ }^{15}$ It is composed of a lactose base decorated with an $\alpha(1-2)$-linked fucose unit at the galactose moiety. In HM of mothers carrying the secretor-gene which accounts for $80 \%$ of the population, ${ }^{16} 2^{\prime}$-FL is one of the most prevalent HMOs. ${ }^{17} 2$ '-FL is recognized as an important HMO for guiding the colonization of bifidobacteria in infants, ${ }^{18}$ as numbers of bifidobacterial species are low in infants fed with HM of non-secretor mothers who lack $\alpha 1$-2-fucosylated HMOs like $2^{\prime}$-FL. ${ }^{16}$ However, kinetics of fermentation of $2^{\prime}$-FL by infant microbiota appears to be more individual specific than fermentation of native inulin. A study by Salli et al. showed that fecal inocula of infants all aged under 9 months could be subdivided into a slow and a fast 2 '-FL fermenting group. ${ }^{19}$ It can be hypothesized that slow fermenters might benefit from combining 2 '-FL with a steadily fermented NDC such as native inulin. Moreover, this combination might ensure saccharolytic fermentation throughout the entire large intestine, ${ }^{20}$ thereby reducing the occurrence of proteolytic fermentation and consequentially preventing detrimental health effects. ${ }^{21}$

Tailoring infant formula with new combinations of NDCs including HMOs might be of interest not only for babies with lower fermentability possibilities but also for example, for infants with different immune backgrounds, like preterm infants who have deviated T-lymphocyte (T-cell) responses. $^{22}$ Naïve T-cells play an important role in the defense against infections. After encountering antigen presenting cells like DCs, the naïve T-cells are activated and differentiate toward antigen specific T-cells. In preterm infants naïve T-cells skew toward a T-helper 2 (Th2) phenotype, while they have a diminished T-helper 1 (Th1) response. ${ }^{22}$ As newborns are exposed to many new antigens, this makes them more susceptible to infections by intracellular pathogens. ${ }^{22}$ Dietary interventions are able to restore the balance between Th1 and Th2 responses. For example, HMOs from preterm milk have been shown to promote Th1 responses and suppress Th2 responses in fetal in vitro organ cultures, ${ }^{23}$ indicating that they contribute to healthier and more balanced T-cell responses. It is unknown whether NDCs have the same effects in healthy and immune compromised infants. It might be possible that for example preterm infants require different NDC formulations than healthy children to restore the balance between Th1 and Th2 responses.

This study had several aims. First we intended to study whether a readily fermentable NDC such as native inulin can influence the fermentation of 2 '-FL in infant microbiota. A second aim was to study whether native inulin in presence and absence of 2'-FL differently modulates dendritic cells and T-cells under normal or Th2 polarized conditions. To this end, we fermented native inulin and the HMO 2'-FL both individually and combined in an in vitro fermentation batch fermentation system using pooled fecal inoculum of 8-week-old infants. We studied the degradation kinetics of the carbohydrates, as well as the changes in microbiota compositions and the production of SCFAs and organic acids over time. To study the effect of the different fermentation digesta on mucosal immune responses, digesta collected at different timepoints during the fermentations were incubated with immature dendritic cells under normal cell culture conditions as well as in presence of the Th2-polarizing cytokines IL-33 or TSLP to simulate the Th2environment in preterm infants. Subsequently, pre-incubated DCs were co-cultured with naïve T-cells to study T-cell skewing.

\section{Materials and methods}

\section{Substrates}

Frutafit IQ (native inulin obtained from chicory roots) was provided by Sensus (Roosendaal, The Netherlands). 2'-FL was provided by Friesland Campina Ingredients (Amersfoort, The Netherlands).

\section{Fermentation of native inulin and $2^{\prime}$-FL by infant fecal inoculum}

Culture medium. Standard ileal efflux medium (SIEM) was prepared as described previously with minor modifications 7 . The SIEM contained low amount of carbohydrates $\left(0.24 \mathrm{~g} \mathrm{~L}^{-1}\right.$ SIEM) to minimize background fermentation. This carbohydrate medium component contained $\left(\mathrm{g} \mathrm{L}^{-1}\right)$ : pectin, 12; xylan, 12; arabinogalactan, 12 amylopectin, 12 and starch, 100. The salt medium component contained $\mathrm{NaCl}\left(9 \mathrm{~g} \mathrm{~L}^{-1}\right)$. The $\mathrm{pH}$ was adjusted to 5.8 using 2 -( $N$-morpholino)ethanesulfonic acid (MES) buffer.

Fecal inoculum. The fecal material from 4 infants $(4 \times$ 0.1 gram) were combined in a pooled fecal inoculum as reported elsewhere, ${ }^{7}$ thereby resembling the bacterial functionality of the different infants. ${ }^{24}$ The infants were exclusively breast-fed, vaginally born and did not have health issues, neither did they receive antibiotic treatment. Parental written informed consent was provided for all infants. Since fecal donations from healthy volunteers are considered as noninvasive, the medical ethical committee approval was not required in the Netherlands. At an age of 8 weeks, the fecal material was collected from the diaper directly after defecation. Subsequently, the fecal material was transferred to tubes and stored at $-80^{\circ} \mathrm{C}$. 
In vitro fermentation. The in vitro fermentations were executed in an anaerobic chamber (gas phase: $4 \% \mathrm{H}_{2}, 15 \% \mathrm{CO}_{2}$ and $81 \% \mathrm{~N}_{2}$ ) (Bactron 300, Sheldon Manufacturing, Cornelius, USA) in duplicate as reported previously. ${ }^{15}$ In short, the pooled fecal inoculum and SIEM containing native inulin and/or 2'-FL were mixed in a ratio of $1: 10(\mathrm{v} / \mathrm{v})$ in sterile flasks with a total volume of $54 \mathrm{ml}$. Native inulin and 2'-FL were fermented individually and in combination in a ratio of $4: 1$. The final concentration of the substrates in the media was $10 \mathrm{mg} \mathrm{ml}^{-1}$ in both individual and combination fermentations. At the start and after 12, 18, 24 and $36 \mathrm{~h}$, samples were collected in triplicate with a syringe. Two samples were heated for $5 \mathrm{~min}$ in a boiling water bath to inactivate enzymes present and subsequently stored at $-20{ }^{\circ} \mathrm{C}$ until further analysis. The other sample was immediately frozen in liquid nitrogen and stored at $-80{ }^{\circ} \mathrm{C}$ to preserve the bacteria. The following control fermentations were included: (1) inoculum without native inulin and 2'-FL to monitor background fermentation, (2) native inulin and 2 '-FL without inoculum to monitor contamination.

\section{Fate of native inulin and 2 '-FL during fermentation}

The degradation of native inulin and 2 '-FL by infant fecal microbiota was monitored by high performance anion exchange chromatography (HPAEC) using a CarboPac PA-1 column (250 mm $\times 2 \mathrm{~mm}$ ID) (Dionex, Sunnyvale, CA, USA) and a CarboPac PA guard column (25 $\mathrm{mm} \times 2 \mathrm{~mm}$ ID) (Dionex) as described elsewhere with minor modification of the eluent profile. ${ }^{6}$ Mobile phase A ( $0.1 \mathrm{M}$ sodium hydroxide) and B (1 M sodium acetate in $0.1 \mathrm{~m}$ sodium hydroxide) were eluted as follows: $0-40 \mathrm{~min}, 0-40 \% \mathrm{~B} ; 40-45 \mathrm{~min}, 40-100 \% \mathrm{~B}$; 45-50 min, washing step with $100 \% \mathrm{~B}$; 50-51 $\mathrm{min}, 100-0 \% \mathrm{~B}$; 51-66 min, equilibration with $100 \% \mathrm{~A}$.

\section{Production of SCFAs and other organic acids during fermentation}

The production of the short-chain fatty acids (SCFAs) acetate, propionate and butyrate, during fermentation of native inulin and 2'-FL was monitored by gas chromatography (GC) as reported elsewhere. ${ }^{7}$ The production of the non-volatile organic acids succinate and lactate, was monitored by high performance liquid chromatography (HPLC) according to the method of Ladirat et al. ${ }^{25}$

\section{Microbial composition analysis}

The DNA extraction followed by polymerase chain reaction (PCR) amplification of the V5-V6 region of 16S ribosomal RNA (rRNA) genes with the unique barcoded primer pair BSF784 (RGGATTAGATACCC) and R1064 (CGACRRCCATGCANACCT), were performed as reported previously. ${ }^{7}$ Ten $\mu$ l of DNA template was used in the triplicate PCR reactions for samples collected at the start of the fermentation which contained 2.3-7 $n g \mathrm{l}^{-1}$ DNA. For samples collected at later timepoints, $0.7 \mu \mathrm{l}$ of DNA template was used. Two synthetic communities with known composition were included as positive controls. ${ }^{26}$

Purification of the PCR products and subsequent preparation for sequencing was performed as reported elsewhere. ${ }^{7}$
In short, $200 \mathrm{ng}$ of each uniquely barcoded sample were combined and subsequently concentrated using the HighPrep PCR kit (MagBio Genomics, Alphen aan den Rijn, The Netherlands) to form an amplicon pool with a volume of $40 \mu$ l. The library was sent for adapter ligation and sequencing with Illumina Hiseq2500 (GATC-Biotech, Konstanz, Germany). Processing and analysis of the 16S rRNA gene amplicon sequencing data were executed using the NG-Tax 2.0 pipeline, with default settings and $\mathrm{R}$ version 3.5.0. ${ }^{27}$ SILVA database release $132^{28}$ was used for taxonomic classification. The functions tax_glom and ordinate in the phyloseq $\mathrm{R}$ package $\mathrm{e}^{29}$ were used to calculate beta-diversity using weighted UniFrac distances based on relative abundance of bacteria at genus level present in the fermentation digesta. The function plot_ordination in the phyloseq $\mathrm{R}_{\text {package }}$ (9) $^{2}$ was used to visualize the output using Principal Coordinate Analysis (PCoA). The contribution of taxa to the principle coordinates was determined from a PCoA plot of the taxa based on the same distances using the function plot_ordination (type:taxa) in the phyloseq $\mathrm{R}$ package. ${ }^{29}$ The function geom_segment in the ggplot2 $\mathrm{R}$ package ${ }^{30}$ was used to plot the taxa vectors with the extracted $x$ - and $y$-coordinates on the first PCoA plot. The sequencing data are available at the European Nucleotide Archive with accession number PRJEB45611.

\section{Dendritic cell and T-cell culture and stimulation under normal conditions or in presence of IL-33 or TSLP}

The immune modulating effects of fermentation digesta of 2'FL and native inulin, either fermented individually or combined, were evaluated using immature DC cytokine responses and naïve T-cell polarization (Fig. 1). DCs generated from umbilical cord blood CD34 + progenitor cells (hematopoietic stem cells) and autologous T-cells were purchased from MatTek Corporation (Ashland, MA, USA). First, DCs were incubated with the digesta for $48 \mathrm{~h}$. Briefly, the DCs were thawed and seeded into 96-well plates at a density of $70 \times 104$ cells per well in a volume of $200 \mu \mathrm{l}$ and cultured overnight $\left(37^{\circ} \mathrm{C}, 21 \%\right.$ $\mathrm{O}_{2}$ and $5 \% \mathrm{CO}_{2}$ ). The next day, the cells were attached to the plates and pre-incubated with normal DC-MM culture medium, or medium containing $50 \mathrm{ng} \mathrm{ml}^{-1} \mathrm{IL}-33$ or TSLP for $1 \mathrm{~h}$. Before replacing the medium by medium containing the fermentation digesta, bacteria were removed from the fermentation digesta by centrifugation (10 min, RT, 12000g).

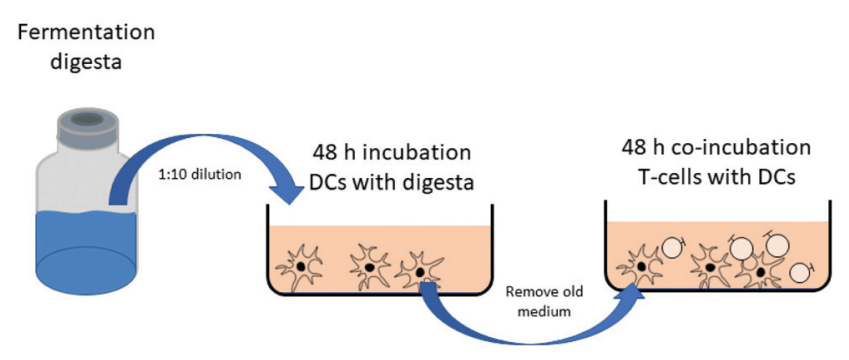

Fig. 1 Design of the in vitro experiments. DCs were incubated with diluted fermentation digesta for $48 \mathrm{~h}$. Subsequently DCs were washed and co-incubated with naïve T-cells in fresh medium. 
Subsequently the supernatants were filtered through a $0.2 \mu \mathrm{m}$ filter, diluted in either normal DC-MM culture medium (MatTek Corporation) containing $50 \mathrm{ng} \mathrm{ml}^{-1}$ polymyxin B (Invivogen, Toulouse, France), or DC-MM culture medium containing $50 \mathrm{ng} \mathrm{ml} \mathrm{m}^{-1}$ Polymyxin B and $50 \mathrm{ng} \mathrm{ml} \mathrm{ml}^{-1}$ IL-33 or ng $\mathrm{ml}^{-1} 50$ TSLP (ImmunoTools GmbH, Friesoythe, Germany) at a ratio of $1: 10$. The $\mathrm{pH}$ was set to 7.4 by the addition of $2 \mathrm{~N}$ $\mathrm{NaOH}$. After $48 \mathrm{~h}$ of incubation with the digesta, cell supernatants were collected and stored at $-20{ }^{\circ} \mathrm{C}$ until further analysis. Subsequently, $300 \mu \mathrm{l}$ fresh TC-MM medium (MatTek Corporation) containing $16 \times 10^{5}$ autologous T-cells was added to selected wells. T-cells were co-incubated with DCs for another $48 \mathrm{~h}$. Afterwards, the supernatants of the co-cultures were collected and stored at $-20{ }^{\circ} \mathrm{C}$ until further analysis. Experiments were repeated 6 times.

\section{Assessment of cytokine expression}

The levels of MCP-1/CCL2, MIP-1 $\alpha /$ CCL3, IL-1 $\beta$, IL-6, IL-10 and TNF $\alpha$ in the DC supernatant and the levels of IFN- $\alpha$, IL-4, IL-5, IL-10, IL-13 and IL-17 in the T-cell supernatant were measured using magnetic Luminex® Assays (R\&D systems, Biotechne, Minneapolis, USA) according to manufacturer's protocol. Briefly, antibody magnetic bead mix were added to 96-well plates. Cytokine standards were re-suspended and serial dilutions were prepared. Standards and samples were added and incubated overnight at $4{ }^{\circ} \mathrm{C}$. After washing, detection antibodies were added and the plates were incubated for $30 \mathrm{~min}$ at room temperature (RT) while shaking. After incubation, the plates were washed again and incubated with streptavidin-PE for $30 \mathrm{~min}$ at RT while shaking. Finally, the plates were washed again and $100 \mu \mathrm{l}$ of wash buffer was added to each well. The plates were analyzed using a Luminex 200 System. The data obtained were analyzed using the Luminex xPONENT software.

\section{Statistical analysis}

Statistical tests were performed using Prism 9 software (GraphPad, San Diego, CA, USA). Outliers were removed after testing using a Grubbs outlier test (alpha $=0.05$ ), which led to the exclusion of 15 out of 1242 samples in our DC cytokine data set. Data was distributed normally and analyzed using a mixed-effects model (REML) to test for differences between groups, followed by a Dunnet's multiple comparisons test to compare fermentation samples of $18 \mathrm{~h}$ and $36 \mathrm{~h}$ with the samples of $0 \mathrm{~h}$ as well as to compare the induced cytokine responses of the digesta of different substrates with each other. Data are shown as averages and error bars represent standard error means.

\section{Results}

\section{Expedited fermentation of 2 '-FL by infant fecal microbiota in presence of native inulin}

To determine if the combination of native inulin and 2 '-FL alters the fermentation behavior of infant fecal microbiota, fermentation digesta of both the individual and combined fer- mentations were analyzed by HPAEC. The fermentation of native inulin by fecal microbiota of 8-week-old infants was size-dependent (Fig. 2A and Fig. S1 $\dagger$ ). Infant fecal microbiota degraded native inulin oligomers with DP $\geq 16$ only to a minor extent. After $36 \mathrm{~h}$ of fermentation $68 \pm 5 \%$ was remaining, whereas native inulin oligomers with DP $\leq 16$ were completely degraded. Although the speed of fermentation was slightly lowered, the extent of fermentation after $36 \mathrm{~h}$ of fermentation did not change significantly when native inulin was fermented in combination with 2'-FL (Fig. 2B). After $36 \mathrm{~h}$ of the combined fermentation $67 \pm 2 \%$ of native inulin oligomers with DP $\geq 16$ was still intact.

The degradation of 2'-FL by fecal microbiota of 8-week-old infants only started after $24 \mathrm{~h}$ of fermentation, but $2^{\prime}$-FL was completely degraded after $36 \mathrm{~h}$ (Fig. 3A). Combining $2^{\prime} \mathrm{FL}$ with native inulin expedited 2'-FL fermentation (Fig. 3B). After $18 \mathrm{~h}$ of fermentation, $16 \pm 1 \%$ of $2^{\prime}$-FL was already degraded by infant fecal microbiota and was completely degraded after 36 hours.

\section{Native inulin and 2 -FL enhance relative abundance of Bifidobacterium}

The fermentation digesta of the individual and combined fermentations were analyzed by $16 \mathrm{~S}$ rRNA gene amplicon sequencing to determine the effect of native inulin and/or 2'-FL on the microbiota composition. The differences in microbiota composition were visualized by PCoA analysis using weighted UniFrac distances with the contribution of the most abundant taxa to PC1 and PC2 presented by taxa vectors (Fig. 4). Together these taxa comprise more than $71 \%$ of each fermentation sample. The relative abundances of all bacterial taxa present in the fermentation samples are shown in Fig. S2. $\dagger$ The fermentation of native inulin by fecal microbiota of 8 -week-old infants enhanced the relative abundance of Bifidobacterium from $46 \pm 2 \%$ at the start of the fermentation to $82 \pm 1 \%$ after $36 \mathrm{~h}$ of fermentation (Fig. 4, Fig. S2, Table S1†). Similar to the control fermentation (Fig. 4), bacteria belonging to the genus Escherichia-Shigella and Enterococcus were enriched upon fermentation, presumably through their stimulation by SIEM medium constituents. ${ }^{31}$

Fermentation of 2'-FL alone by infant fecal microbiota resulted in a less pronounced increase in relative abundance of Bifidobacterium as observed with native inulin fermentation (Fig. 4, Fig. S2, Table S1 $\dagger$ ). After a decrease in relative abundance in the first $18 \mathrm{~h}$ of fermentation from $46 \pm 1 \%$ to $32 \pm$ $0.5 \%$, we observed that after 36 h $64 \pm 3 \%$ of the bacteria belonged to the genus Bifidobacterium. The initial decrease in relative abundance of Bifidobacterium is suggested to be caused by the fast growth of bacteria (Escherichia-Shigella and Enterococcus) on SIEM medium components in this initial stage. This phenomenon was also observed in our previous study with identical fermentation set-up where the total $16 \mathrm{~S}$ rRNA gene copy numbers increased with a factor of 2000 in the first $14 \mathrm{~h}$ of a control fermentation without added NDCs. ${ }^{6}$

The fermentation of the combination of native inulin and 2'-FL resembled the effects of native inulin fermentation 
A. native inulin - individual

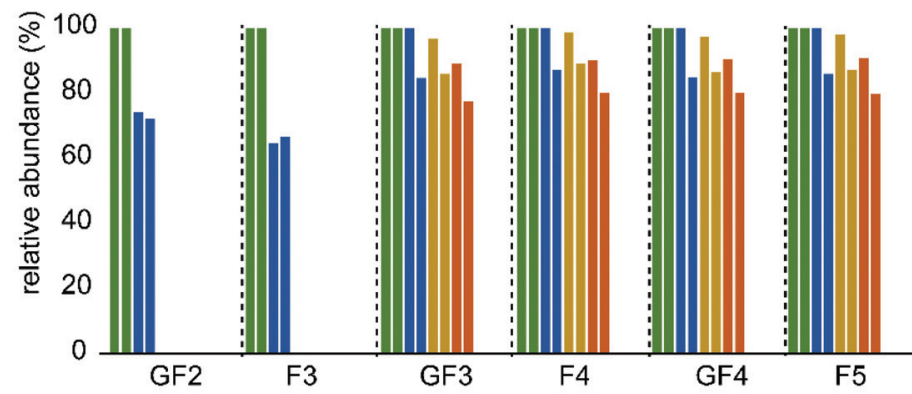

B. native inulin - combination with 2'-FL

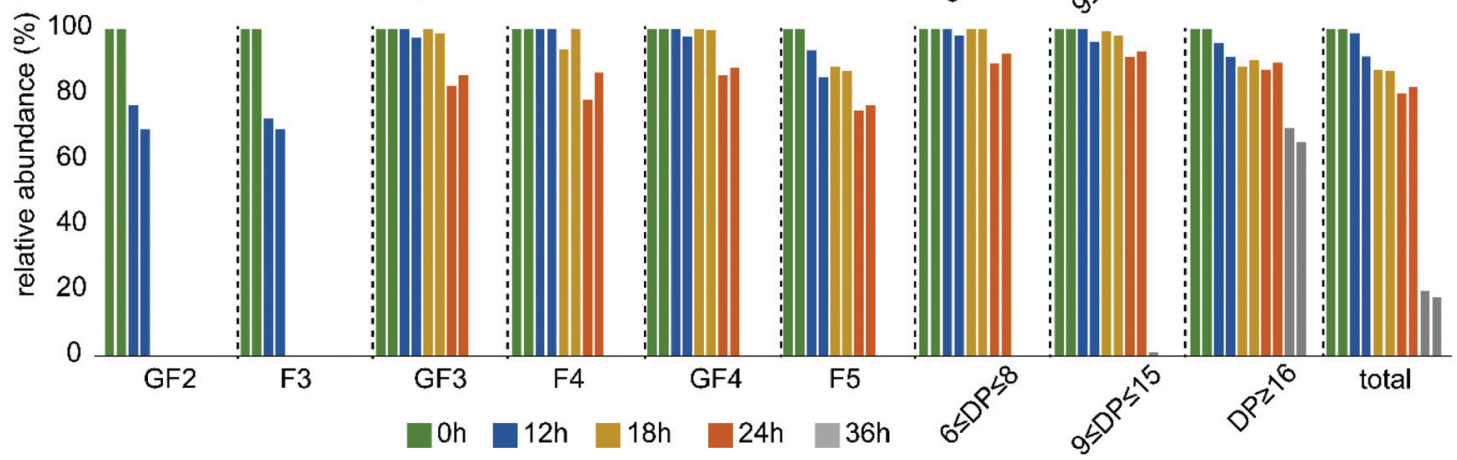

Fig. 2 Relative abundance of compounds with different DP belonging to the GF or F series (glucose or fructose moiety at the reducing end) present in native inulin in fermentation digesta collected at the start and after 12, 18, 24 and $36 \mathrm{~h}$ of in vitro fermentation using pooled fecal inoculum of 8-week-old infants, individual (A) and combined with 2'-FL (B). Fermentations were performed in duplicate with each individual series presented individually. Analysis was performed by HPAEC-PAD. Concentrations per compound/DP range in the original native inulin mixture were set to $100 \%$.

A. 2'-FL - individual

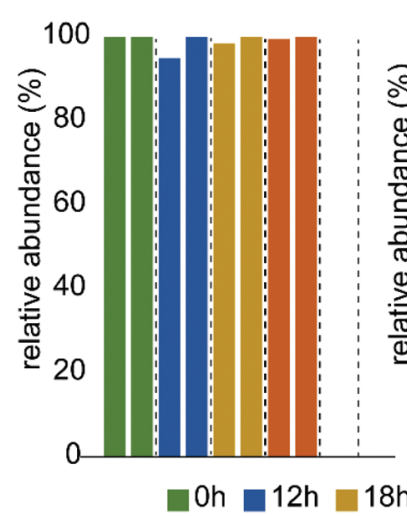

B. 2'-FL - combination with native inulin

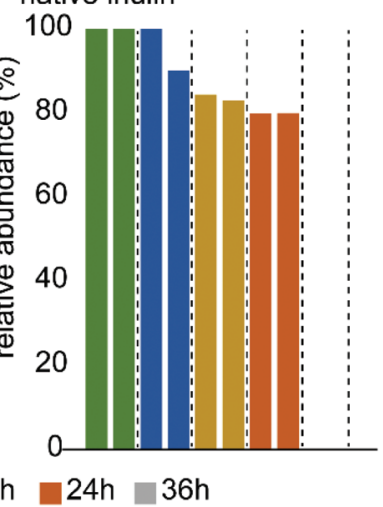

Fig. 3 Relative abundance of 2'-FL in fermentation digesta collected at the start and after 12,18, 24 and 36 of in vitro fermentation using pooled fecal inoculum of 8 -week-old infants, individual (A) and combined with native inulin (B). Fermentations were performed in duplicate with each individual series presented individually. Analysis was performed by HPAEC-PAD.

alone. After $36 \mathrm{~h}$ of fermentation, $80 \pm 1 \%$ of the bacteria belonged to the genus Bifidobacterium (Fig. 4, Fig. S2, Table $\mathrm{S} 1 \dagger$ ). In addition, background fermentation on SIEM

medium constituents by bacteria belonging to the genus Escherichia-Shigella and Enterococcus was observed.

\section{Acetate and lactate production during fermentation of native} inulin and 2 -FL by infant fecal microbiota

The production of the immune-active organic acids acetate, propionate, butyrate as well as lactate and succinate ${ }^{12,32}$ by fecal microbiota of 8 -week-old infants was monitored during the fermentation of native inulin and/or 2'-FL. Butyrate was not detected in any of the fermentation digesta. Compared to the control fermentation without added NDCs $(2.3 \pm 0.1 \mu \mathrm{mol}$ $\mathrm{mg}^{-1}$ after $36 \mathrm{~h}$ of fermentation), the fermentation of native inulin by fecal microbiota of 8-week-old infants resulted in significant production of organic acids after $36 \mathrm{~h}$ (Fig. 5). After $18 \mathrm{~h}$ of fermentation, a total organic acid level of $2.9 \mu \mathrm{mol}$ $\mathrm{mg}^{-1}$ was reached, with a strong increase up to $9.8 \mu \mathrm{mol} \mathrm{mg}{ }^{-1}$ after $36 \mathrm{~h}$ of fermentation. The ratio of the produced acetate, lactate, succinate and propionate was $73: 23: 3: 1$.

In comparison to native inulin, the fermentation of 2 '-FL by infant fecal microbiota resulted in higher organic acid production after $36 \mathrm{~h}$ (Fig. 5). However, after $18 \mathrm{~h}$ of fermentation, a total organic acid level of only $2.1 \mu \mathrm{mol} \mathrm{mg}{ }^{-1}$ was reached, which sharply increased up to $11.9 \mu \mathrm{mol} \mathrm{mg}^{-1}$ after $36 \mathrm{~h}$ of fermentation. The ratio of the produced acetate, lactate, succinate and propionate was $65: 29: 4: 2$. 


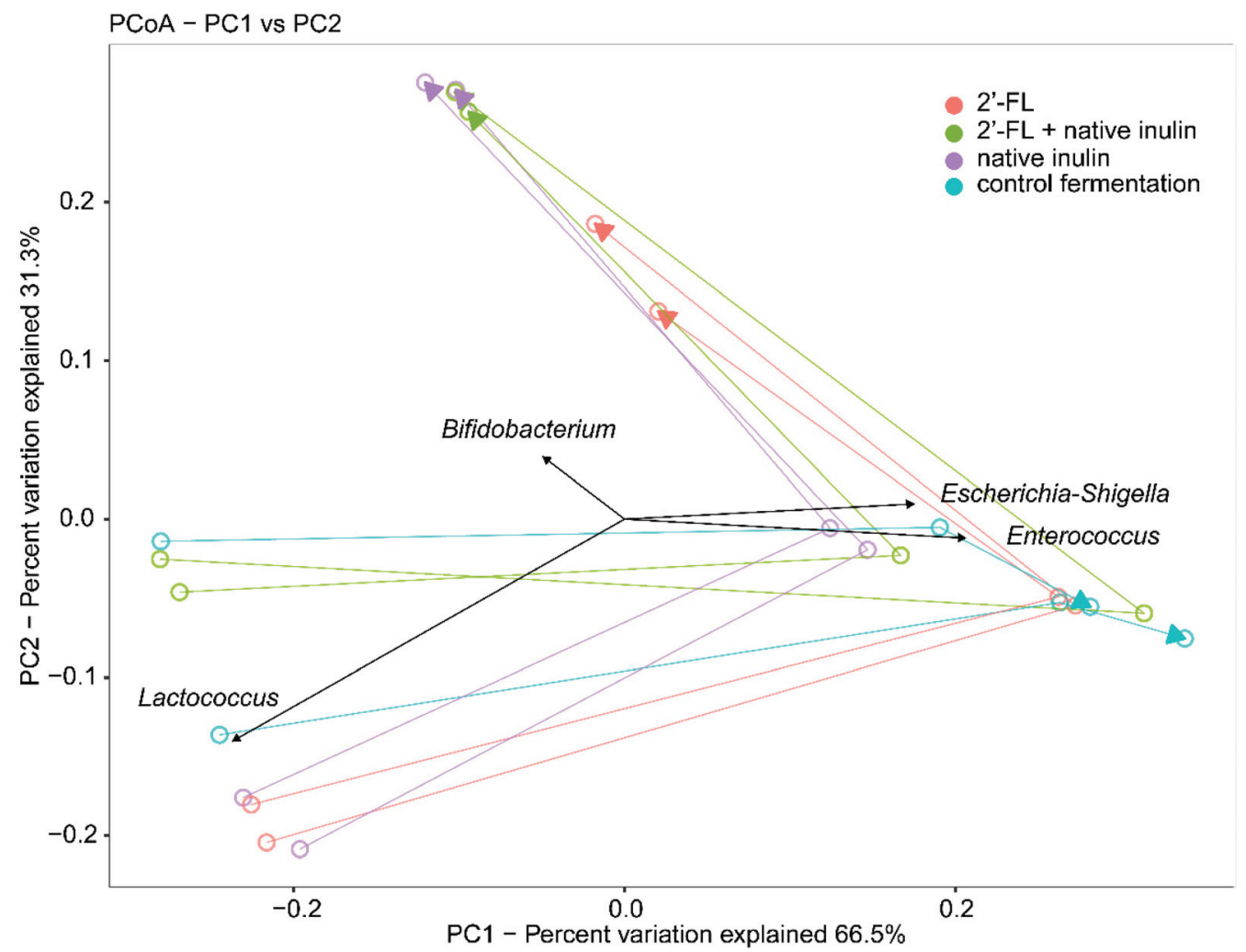

Fig. 4 PCoA using weighted UniFrac distances of microbiota composition in fermentation digesta collected at the start and after 18 and $36 \mathrm{~h}$ of fermentation of native inulin, 2'-FL, native inulin/2'-FL or the control, using pooled fecal inoculum of 8-week-old infants. Direction of changes in time is presented by arrows in the same color. The contribution of selected taxa to PC1 and PC2 is presented by the direction and length of the taxa arrows (black). Fermentations were performed in duplicate with each individual series presented individually.

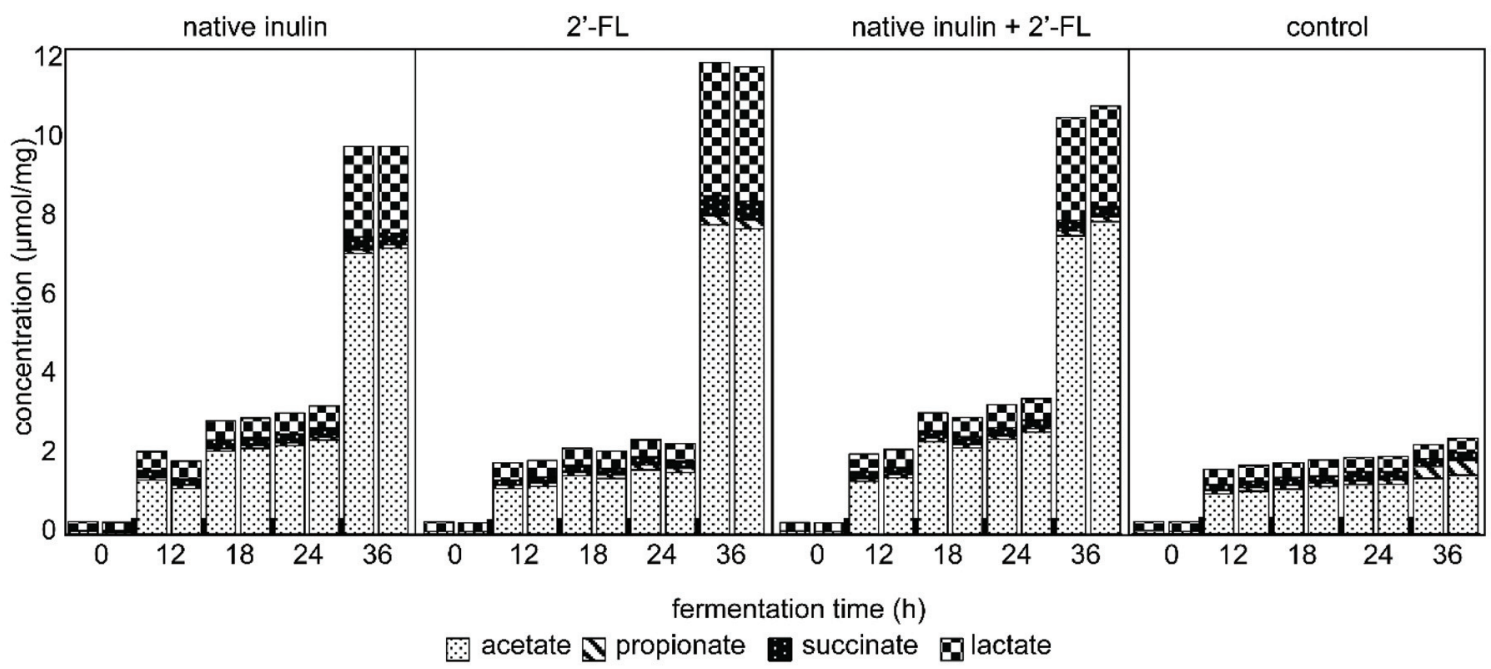

Fig. 5 Concentration of the SCFAs acetate and propionate as well as the organic acids lactate and succinate in fermentation digesta collected at the start and after 12, 18, 24 and $36 \mathrm{~h}$ from in vitro fermentation of native inulin, 2'-FL, native inulin/2'-FL or the control, using pooled fecal inoculum of 8 -week-old infants. Fermentations were performed in duplicate with each individual series presented individually.

The organic acid production after $36 \mathrm{~h}$ of the fermentation of the combination of native inulin and $2^{\prime}$-FL was the average of the individual fermentations (Fig. 5). After $18 \mathrm{~h}$ of fermentation, a total organic acid level of $3 \pm 0.1$ $\mu \mathrm{mol} \mathrm{m \textrm {m } ^ { - 1 }}$ was reached, which further increased up to $10.7 \pm 0.1 \mu \mathrm{mol} \mathrm{mg}{ }^{-1}$ after $36 \mathrm{~h}$ of fermentation. The ratio of 
the produced acetate, lactate, succinate and propionate was $72: 24: 3: 1$

Fermentation digesta of 2 '-FL and native inulin differently attenuate cytokine responses in dendritic cells under normal conditions and in presence of the Th2-associated cytokines IL-33 and TSLP

DCs are abundantly present under the epithelial lining of the GI tract and can come in contact with the luminal content. ${ }^{33}$ As DCs are important initiators of mucosal immune responses, ${ }^{34}$ we investigated the effect of the digesta of native inulin, 2'-FL and native inulin/2'-FL fermented with pooled fecal inoculum of 8-week-old infants on DC cytokine secretion (Fig. 6).

Under normal culture conditions, the digesta of the blank fermentation induced fermentation time dependent cytokine responses. Incubation of DCs with the $t=18$ digesta of the fermentation of native inulin resulted in a significant higher level of IL-6, while incubation with the $t=36$ digesta of this fermentation resulted in significant higher levels of IL-1 $\beta$, IL-6 and TNF $\alpha$ and a significant lower level of IL-10 when compared to the corresponding digesta in time of the blank fermentation. Incubation of DCs with the $t=18$ digesta of the 2 '-FL fermentation resulted in a significant higher level of IL- 6 when compared to the blank. In contrast, cytokine responses induced by the $t=$ 36 digesta and both the $t=18$ and $t=36$ digesta of the native inulin/2'-FL fermentation did not differ from the cytokine responses induced by the corresponding digesta of the blank fermentation. When cytokine responses between different groups were compared, incubation of DCs with the $t=18$ digesta of 2 '-FL resulted in significantly higher TNF $\alpha$ levels than the $t=18$ digesta of native inulin/2'-FL. Incubation with the $t=$ 36 digesta of 2 '-FL resulted in significant higher levels of IL-1 $\beta$ and IL- 6 when compared to the $t=36$ digesta of native inulin.

DCs were also incubated with fermentation digesta in presence of the Th2-polarizing cytokines IL-33 and TSLP. Incubation of DCs with the $t=18$ and $t=36$ fermentation digesta of the blank in presence of IL-33 or TSLP gave profound differences in response in a fermentation time dependent manner (Fig. 5). In presence of IL-33, incubation of DCs with the $t=36$ digesta of the native inulin/2'-FL fermentation resulted in a significant higher level of IL-1 $\beta$ when compared to the $t=36$ digesta of the blank fermentation. Compared to incubation under normal culture conditions, incubation of DCs in presence of IL-33 only resulted in a significant higher IL-1 $\beta$ level with the $t=18$ digesta of the blank fermentation.

In presence of TSLP, incubation of DCs with the $t=36$ digesta of the 2'-FL fermentation resulted in significant higher levels of $\mathrm{TNF} \alpha$ when compared to the $t=36$ digesta of native inulin. In addition, incubation of DCs with the $t=36$ digesta of native inulin resulted in significant higher levels of CCL2/ MCP-1 when compared to the $t=36$ digesta of the native inulin/2'-FL fermentation. Compared to incubation under normal culture conditions, the $t=18$ digesta of the blank fermentation induced higher levels of IL- $1 \beta$ and the $t=18$ digesta of the native inulin fermentation induced higher levels of
TNF $\alpha$. In addition, the $t=36$ digesta of the native inulin fermentation resulted in higher levels of IL-1 $\beta$ and IL-10 and the $t$ $=36$ digesta of the 2 '-FL fermentation induced lower levels of TNF $\alpha$. Incubation with the $t=18$ digesta of the native inulin/ 2 '-FL fermentation in presence of TSLP resulted in higher levels of IL-1 $\beta$ and IL-10. Incubation of DCs with IL-33 or TSLP alone had no significant effect on basal cytokine responses when compared to untreated DCs cultured in normal cell culture (Fig. S3†).

\section{T-cell responses are influenced by 2 '-FL and native inulin fermentation digesta}

T-cells play an important role in the regulation of mucosal immune reactions in response to food components. ${ }^{35}$ To investigate whether the fermentation digesta could influence T-cell cytokine production, DCs that were pre-incubated with fermentation digesta, under normal conditions or in presence of the Th2-polarizing cytokines IL-33 or TSLP, were co-cultured with naïve T-cells and cytokine levels in the supernatant were measured. Experiments were only done with fermentation digesta of the native inulin/2'-FL combination fermentation and compared to the samples of the blank fermentation.

Co-culturing of T-cells with DCs that were pre-incubated with the $t=0$ or $t=36$ digesta of the blank fermentation resulted in the secretion of IL-4, IL-5, IL-10 and IL-13 (Fig. 7). The cytokines IL-17A and IFN $\alpha$ were not detected in the cell supernatants. Under normal conditions, no significant differences were found in cytokine secretion by T-cells co-cultured with DCs that were pre-incubated with the $t=0$ and $t=36$ digesta of the blank fermentation, neither with $t=0$ or $t=36$ digesta of the native inulin/2'-FL fermentation. However, the level of IL-4 secretion by T-cells that were co-cultured with DCs was significantly lower when pre-incubated with $t=36$ digesta of the blank fermentation than when pre-incubated with the $t$ $=36$ digesta of the native inulin $/ 2^{\prime}$-FL fermentation.

Co-culturing T-cells with DCs that were pre-incubated with the $t=36$ digesta of the blank fermentation in presence of IL-33 resulted in significant lower levels of IL-4 when compared to T-cells that were co-cultured with DCs that were preincubated with the $t=0$ digesta of the same fermentation. In addition, IL-4 secretion was higher for T-cells that were co-cultured with the $t=36$ digesta of the native inulin/2'-FL fermentation in presence of IL-33 than for the corresponding T-cells that were co-cultured with the $t=36$ digesta of the blank fermentation.

Co-culturing of T-cells with DCs that were pre-incubated with the $t=36$ digesta of the blank fermentation in presence of TSLP resulted in significant lower levels of IL-4 when compared to T-cells that were co-cultured with DCs that were preincubated with the $t=0$ digesta of the same fermentation.

\section{Discussion}

The quality of infant nutrition is an important factor for guiding the gut microbiota colonization and for supporting 

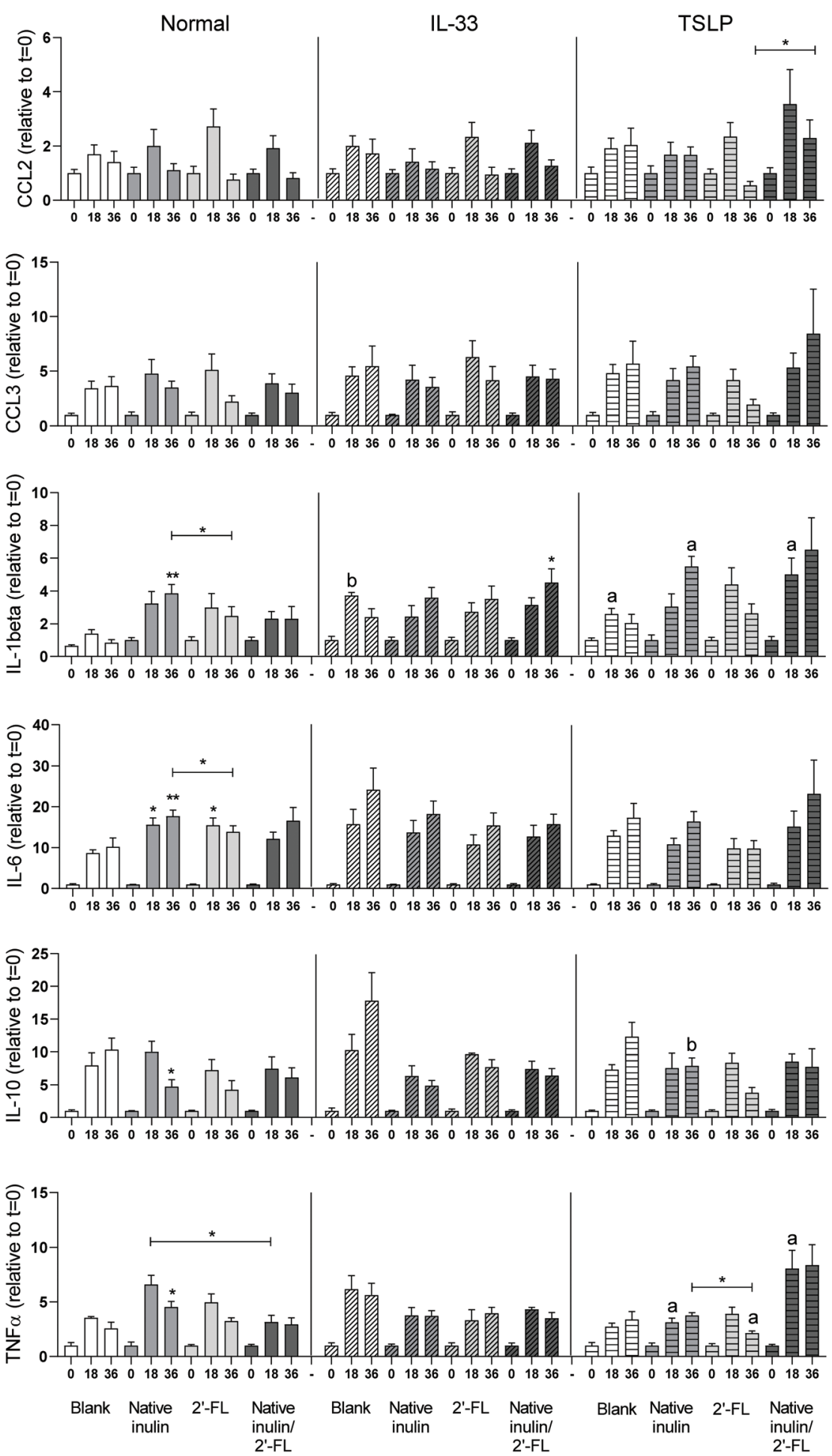

Fig. 6 Cytokine secretion by immature dendritic cells when stimulated with digesta of the blank fermentation and fermentations of native inulin, $2^{\prime}-\mathrm{FL}$ or the combination of native inulin/2'-FL. Data is expressed as mean with SEM. Stars above bars represent statistical different response compared to the corresponding blank samples $\left({ }^{*} p<0.05,{ }^{* *} p<0.01,{ }^{* *} p<0.001\right)$. Statistical differences between cytokine responses induced under normal conditions or in presence of IL-33 or TSLP are indicated on the right side of the graph $\left({ }^{\mathrm{a}} p<0.05,{ }^{\mathrm{b}} p<0.01\right)$.

the development of healthy mucosal immune responses in infants. ${ }^{36}$ NDCs including native inulin and the HMO 2'-FL are often added to cow-milk based infant formulas to mimic the prebiotic and immune properties of HMOs present in human milk. ${ }^{36}$ However, to date only limited knowledge is available on the impact of the combination of NDCs on these health- 

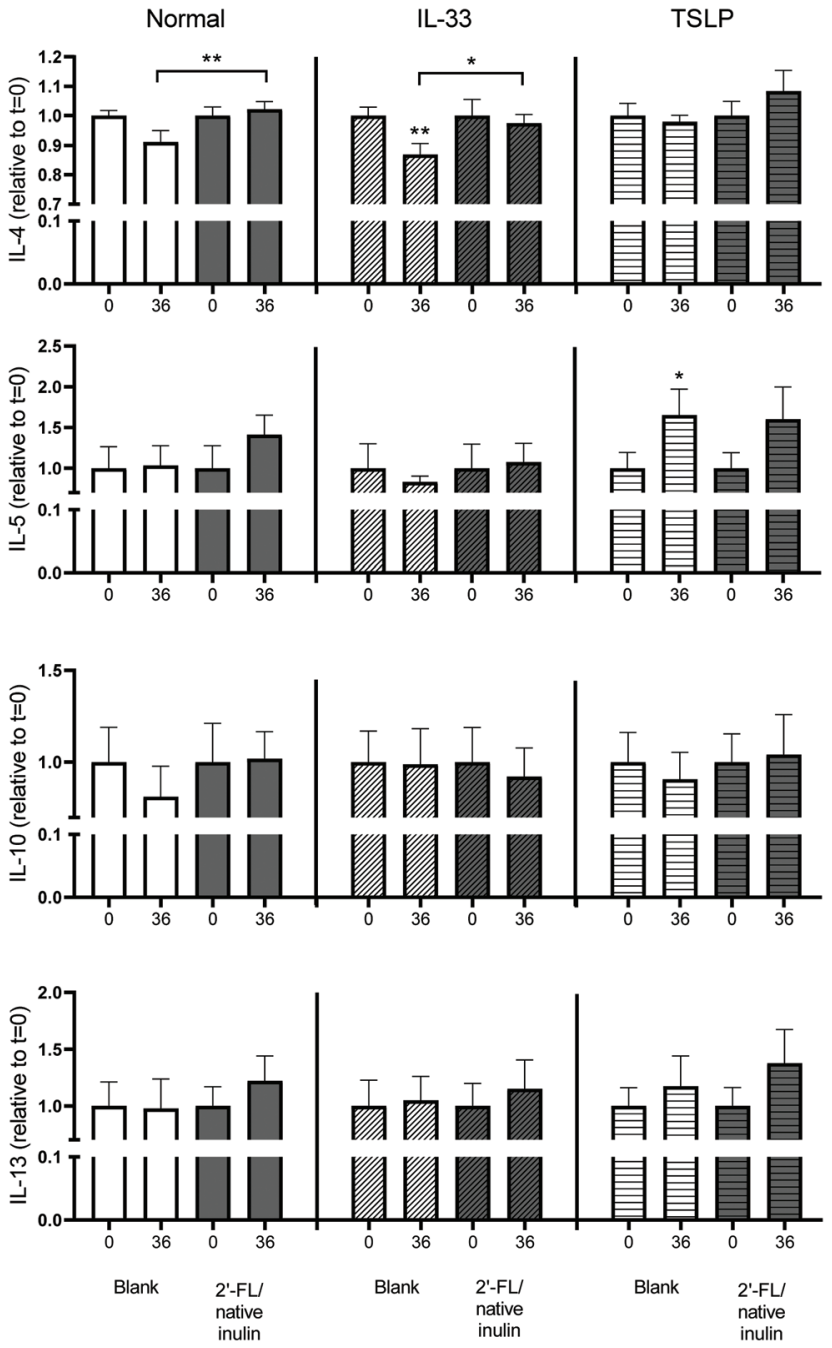

Fig. 7 Cytokine secretion by T-cells that were co-cultured with DCs that were pre-incubated with digesta of the blank fermentation or the native inulin/2'-FL fermentation under normal conditions or in presence of the Th2-polarizing cytokines IL-33 or TSLP. Data is expressed as mean with SEM. Stars above bars represent statistical different responses between groups $\left({ }^{*} p<0.05,{ }^{* *} p<0.01,{ }^{* *} p<0.001\right)$.

beneficial properties. In addition, minor knowledge is available about the immune effects of NDCs in infants with different immune backgrounds. For this reason, we investigated whether combining native inulin and 2'-FL influenced the fermentation kinetics of these NDCs. In addition, we studied the effect of the fermentation digesta on DC and T-cell responses under normal cell culture conditions as well as in presence of the Th2-polarizing cytokines IL-33 or TSLP.

The native inulin was fermented in a size-dependent fashion by the fecal microbiota of 8-week-old infants, which is in line with the observations in our previous study. ${ }^{6}$ Native inulin with $\mathrm{DP} \leq 16$ was primarily degraded by the infant fecal microbiota with a clear preference for the DP3 oligomers. Fermentation of native inulin in combination with $2^{\prime}$-FL did not substantially affect the degradation kinetics of native inulin. In contrast, the degradation of 2 -FL was expedited when fermented in combination with native inulin. The enhanced fermentability of 2 '-FL might be explained by the early stimulation of the fecal microbiota through the fermentation of native inulin, which increased the expression of $\beta$-fructofuranosidase required for the degradation of native inulin ${ }^{37}$ and, at the same time, expansion of the total amount and/or variety of 2'-FL-degrading enzymes expressed by the infant fecal microbiota.

The fermentation of both native inulin and $2^{\prime} \mathrm{FL}$ by fecal microbiota of 8-week-old infants increased the relative abundance of Bifidobacterium, which coincided with the production of acetate and lactate. These organic acids are important end products of the bifid shunt, which is a unique hexose metabolism pathway of Bifidobacterium. ${ }^{38}$ The stimulation of Bifidobacterium by native inulin and 2 -FL corroborates the findings of previous in vitro ${ }^{6,18,19,39}$ and in vivo ${ }^{16,40-42}$ studies. Although the fermentability of 2'-FL was enhanced when combined with native inulin, no synergistic effects were observed with respect to the bifidobacterial stimulation and organic acid production during the fermentation of the combination of native inulin and 2 '-FL.

The fermentation digesta of the native inulin and $2^{\prime}$-FL, either fermented alone or combined, induced significant different cytokine secretion patterns in DCs cultured under normal conditions when compared to DCs incubated with digesta of blank fermentation. These differences can possibly be explained by the changes in microbiota composition and the production of SCFAs and other bacterial metabolites during the fermentation of the NDCs. Compared to the blank fermentation, fermentation of all NDCs resulted in a high relative abundance of Bifidobacterium and the production of acetate and lactate. Bacterial products from Bifidobacteria, such as exopolysaccharides, ${ }^{43}$ bacterial RNA/DNA and lipoproteins ${ }^{44}$ can interact with DCs via specialized immune receptors including Toll like receptors (TLRs) and C-type lectin receptors expressed on the cell surface. ${ }^{44,45}$ SCFAs have been shown to influence DC cytokine production via interaction with GPCRs receptors including GPR43, GPR41 and GPR109A. ${ }^{32}$ We did not find significant differences in cytokine responses between DCs incubated with digesta of the different NDC fermentations i.e., native inulin and 2'-FL either alone or combined, which could be explained by a considerable overlap in changes in microbiota composition and SCFA production during the different fermentations.

Interestingly, our data suggest that the immune background of infants is influencing the impact of the NDCs as the DC immune responses were different under normal conditions or when incubated in presence of the Th2-polarizing cytokines IL-33 or TSLP. ${ }^{46}$ A very pronounced effect was observed for IL-1 $\beta$ and TNF $\alpha$ secretion by DCs when incubated with the fermentation digesta in presence of the Th2-polarizing cytokine TSLP, which is linked to many allergic diseases. ${ }^{47}$ In the in vivo situation, TSLP is primarily secreted by epithelial cells and plays an important role in the maturation of T-cells through the activation of DCs. Hence, the increase in IL-1 $\beta$ and TNF $\alpha$ secretion upon incubation with the fermentation digesta in 
presence of TSLP might indicate a stronger immune response in TSLP related diseases which includes asthma and allergic inflammation. ${ }^{47}$

Furthermore, pre-incubation of DCs with digesta of the native inulin/2'-FL fermentation significantly impacted T-cell cytokine responses. Pre-incubation of DCs with the $t=36$ sample of the native inulin/2'-FL combination fermentation resulted in a higher production of the Th2-skewing cytokine IL- $4^{48}$ in T-cells under both normal and IL-33 culture conditions. Interestingly, this difference was not observed for T-cells incubated with DCs that were pre-incubated with the digesta in presence of TLSP, indicating that the immune background of infants also influences the outcome of T-cell responses. Taken together, our results suggest that immune compromised infants might benefit from infant formulas with different and more tailored NDC formulations than healthy infants, which can possibly be accomplished by making new combinations of NDCs.

\section{Conclusions}

This study provides insight in the effect of combining different NDCs and the influence of their fermentation digesta on DC and T-cell cytokine responses under different culture conditions. Our data shows that the fermentability of slowly-fermentable 2 '-FL could be accelerated by combining it with the more rapid-fermentable native inulin. The fermentation digesta of the different fermentations were also shown to influence DC and T-cell cytokine responses differently under normal and Th2-polarized culture conditions, indicating that NDCs might have different effects in immune compromised infants. Our findings might contribute to the design and development of tailored NDC mixtures for the application in infant formulas for infants with different immune backgrounds.

\section{Author contributions}

R.A., M.J.L, H.A.S. and P.D.V. designed the study. M.J.L performed the fermentation experiments. R.A. and M. B. performed the cell-based experiments. M.J.L performed the microbiota analysis, supported by E.Z., M.M.F. and B.J.D. H. assisted with the cell-based experiments. R.A. M.J.L, H.A.S. and P.D.V. wrote the manuscript. All authors have revised and improved the manuscript.

\section{Conflicts of interest}

There are no conflicts to declare.

\section{Acknowledgements}

Research of Renate Akkerman and Madelon J. Logtenberg was performed in the public-private partnership 'CarboKinetics' coordinated by the Carbohydrate Competence Center (CCC, http://www.cccresearch.nl). CarboKinetics is financed by participating industrial partners Agrifirm Innovation Center B.V., Cooperatie Avebe U.A., DSM Food Specialties B.V., FrieslandCampina Nederland B.V., Nutrition Sciences N.V., VanDrie Holding N.V. and Sensus B.V., and allowances of the Dutch Research Council (NWO). The authors would like to thank Ineke Heikamp-de Jong for her support during the sequencing preparations.

\section{References}

1 L. Bode, Human milk oligosaccharides: every baby needs a sugar mama, Glycobiology, 2012, 22, 1147-1162.

2 L. Cheng, R. Akkerman, C. Kong, M. T. C. Walvoort and P. de Vos, More than sugar in the milk: human milk oligosaccharides as essential bioactive molecules in breast milk and current insight in beneficial effects, Crit. Rev. Food Sci. Nutr., 2020, 1-17, DOI: 10.1080/10408398.2020.1754756.

3 G. Kelly, Inulin-type prebiotics-a review: part 1, Altern. Med. Rev., 2008, 13, 315-329.

4 J. Knol, P. Scholtens, C. Kafka, J. Steenbakkers, S. Gro, K. Helm, M. Klarczyk, H. Schöpfer, H. M. Böckler and J. Wells, Colon microflora in infants fed formula with galacto- and fructo-oligosaccharides: more like breast-fed infants, J. Pediatr. Gastroenterol. Nutr., 2005, 40, 36-42.

5 Y. Zhu, L. Wan, W. Li, D. Ni, W. Zhang, X. Yan and W. Mu, Recent advances on 2'-fucosyllactose: physiological properties, applications, and production approaches, Crit. Rev. Food Sci. Nutr., 2020, 1-10, DOI: 10.1080/ 10408398.2020.1850413.

6 M. J. Logtenberg, R. Akkerman, R. An, G. D. A. Hermes, B. J. de Haan, M. M. Faas, E. G. Zoetendal, H. A. Schols and P. de Vos, Fermentation of Chicory FructoOligosaccharides and Native Inulin by Infant Fecal Microbiota Attenuates Pro-Inflammatory Responses in Immature Dendritic Cells in an Infant-Age-Dependent and Fructan-Specific Way, Mol. Nutr. Food Res., 2020, 64, 2000068.

7 R. Akkerman, M. J. Logtenberg, R. An, M. A. Van Den Berg, B. J. de Haan, M. M. Faas, E. Zoetendal, P. de Vos and H. A. Schols, Endo-1,3(4)- $\beta$-Glucanase-Treatment of Oat $\beta$-Glucan Enhances Fermentability by Infant Fecal Microbiota, Stimulates Dectin-1 Activation and Attenuates Inflammatory Responses in Immature Dendritic Cells, Nutrients, 2020, 12, 1660.

8 L. Cheng, M. B. G. Kiewiet, A. Groeneveld, A. Nauta and P. de Vos, Human milk oligosaccharides and its acid hydrolysate LNT2 show immunomodulatory effects via TLRs in a dose and structure-dependent way, J. Funct. Foods, 2019, 59, 174-184.

9 L. Vogt, U. Ramasamy, D. Meyer, G. Pullens, K. Venema, M. M. Faas, H. A. Schols and P. de Vos, Immune modulation by different types of beta2->1-fructans is toll-like receptor dependent, PLoS One, 2013, 8, e68367. 
10 H. Ito, N. Takemura, K. Sonoyama, H. Kawagishi, D. L. Topping, M. A. Conlon and T. Morita, Degree of polymerization of inulin-type fructans differentially affects number of lactic acid bacteria, intestinal immune functions, and immunoglobulin A secretion in the rat cecum, J. Agric. Food Chem., 2011, 59, 5771-5778.

11 N. T. Baxter, A. W. Schmidt, A. Venkataraman, K. S. Kim, C. Waldron and T. M. Schmidt, Dynamics of human gut microbiota and short-chain fatty acids in response to dietary interventions with three fermentable fibers, mBio, 2019, 10, e02566-e02518.

12 P. M. Smith, M. R. Howitt, N. Panikov, M. Michaud, C. A. Gallini, M. Bohlooly-Y, J. N. Glickman and W. S. Garrett, The microbial metabolites, short-chain fatty acids, regulate colonic treg cell homeostasis, Science, 2013, 341, 569-573.

13 G. A. Sprenger, F. Baumgärtner and C. Albermann, Production of human milk oligosaccharides by enzymatic and whole-cell microbial biotransformations, J. Biotechnol., 2017, 258, 79-91.

14 F. Baumgärtner, L. Seitz, G. A. Sprenger and C. Albermann, Construction of Escherichia coli strains with chromosomally integrated expression cassettes for the synthesis of 2'-fucosyllactose, Microb. Cell Fact., 2013, 12, 40.

15 C. Kong, M. M. Faas, P. de Vos and R. Akkerman, Impact of dietary fibers in infant formulas on gut microbiota and the intestinal immune barrier, Food Funct., 2020, 11, 94459467.

16 Z. T. Lewis, S. M. Totten, J. T. Smilowitz, M. Popovic, E. Parker, D. G. Lemay, M. L. Van Tassell, M. J. Miller, Y.-S. Jin, J. B. German, C. B. Lebrilla and D. A. Mills, Maternal fucosyltransferase 2 status affects the gut bifidobacterial communities of breastfed infants, Microbiome, 2015, 3, 1-21.

17 N. Sprenger, L. Y. Lee, C. A. De Castro, P. Steenhout and S. K. Thakkar, Longitudinal change of selected human milk oligosaccharides and association to infants' growth, an observatory, single center, longitudinal cohort study, PLoS One, 2017, 12, e0171814.

18 P. Van den Abbeele, C. Duysburgh, E. Vazquez, J. Chow, R. Buck and M. Marzorati, 2'-Fucosyllactose alters the composition and activity of gut microbiota from formula-fed infants receiving complementary feeding in a validated intestinal model, J. Funct. Foods, 2019, 61, 103484.

19 K. Salli, H. Anglenius, J. Hirvonen, A. A. Hibberd, I. Ahonen, M. T. Saarinen, K. Tiihonen, J. Maukonen and A. C. Ouwehand, The effect of 2'-fucosyllactose on simulated infant gut microbiome and metabolites; a pilot study in comparison to GOS and lactose, Sci. Rep., 2019, 9, 13232.

20 S. Shim, J. Verdonk, W. Pellikaan and W. Verstegen, Differences in microbial activities of faeces from weaned and unweaned pigs in relation to in vitro fermentation of different sources of inulin-type oligofructose and pig feed ingredients, Asian-Australas. J. Anim. Sci., 2007, 20, 1444-1452.
21 K. Korpela, Diet, microbiota, and metabolic health: Tradeoff between saccharolytic and proteolytic fermentation, Annu. Rev. Food Sci. Technol., 2018, 9, 65-84.

22 T. Sproat, R. P. Payne, N. D. Embleton, J. Berrington and S. Hambleton, T Cells in preterm infants and the influence of milk diet, Front. Immunol., 2020, 11, 1035.

23 Y. He, S. Liu, S. Leone and D. S. Newburg, Human colostrum oligosaccharides modulate major immunologic pathways of immature human intestine, Mucosal Immunol, 2014, 7, 1326-1339.

24 M. J. Logtenberg, J. C. M. Vink, R. M. Serierse, R. An, G. D. A. Hermes, H. Smidt and H. A. Schols, Pooled faecal inoculum can predict infant fiber fermentability despite high inter-individual variability of microbiota composition, Bioact. Carbohydr. Diet. Fibre, 2020, 100235.

25 S. E. Ladirat, F. H. Schuren, M. H. Schoterman, A. Nauta, H. Gruppen and H. A. Schols, Impact of galacto-oligosaccharides on the gut microbiota composition and metabolic activity upon antibiotic treatment during in vitro fermentation, FEMS Microbiol. Ecol., 2014, 87, 41-51.

26 J. Ramiro-Garcia, G. D. A. Hermes, C. Giatsis, D. Sipkema, E. G. Zoetendal, P. J. Schaap and H. Smidt, NG-Tax, a highly accurate and validated pipeline for analysis of $16 \mathrm{~S}$ rRNA amplicons from complex biomes, F1000Research, 2016, 5, 1791-1791.

27 W. Poncheewin, G. D. A. Hermes, J. C. J. van Dam, J. J. Koehorst, H. Smidt and P. J. Schaap, NG-Tax 2.0: A semantic framework for high-throughput amplicon analysis, Front. Genet., 2020, 10, 1366.

28 C. Quast, E. Pruesse, P. Yilmaz, J. Gerken, T. Schweer, P. Yarza, J. Peplies and F. O. Glöckner, The SILVA ribosomal RNA gene database project: improved data processing and web-based tools, Nucleic Acids Res., 2012, 41, D590D596.

29 P. J. McMurdie and S. Holmes, Phyloseq: an R package for reproducible interactive analysis and graphics of microbiome census data, PLoS One, 2013, 8, e61217.

30 H. Wickham, ggplot2: Elegant graphics for data analysis, Springer-Verlag, New York, 2016, ISBN: 978-3-319-24277-4.

31 A. J. Richardson, N. McKain and R. J. Wallace, Ammonia production by human faecal bacteria, and the enumeration, isolation and characterization of bacteria capable of growth on peptides and amino acids, BMC Microbiol., 2013, 13, 6.

32 C. Nastasi, M. Candela, C. M. Bonefeld, C. Geisler, M. Hansen, T. Krejsgaard, E. Biagi, M. H. Andersen, P. Brigidi, N. Ødum, T. Litman and A. Woetmann, The effect of short-chain fatty acids on human monocytederived dendritic cells, Sci. Rep., 2015, 5, 16148.

33 H. Tezuka and T. Ohteki, Regulation of IgA Production by Intestinal Dendritic Cells and Related Cells, Front. Immunol., 2019, 10, 1891.

34 J. L. Owen and M. Mohamadzadeh, Microbial activation of gut dendritic cells and the control of mucosal immunity, J. Interferon Cytokine Res., 2013, 33, 619-631. 
35 F. van Wijk and $H$. Cheroutre, Intestinal T cells: facing the mucosal immune dilemma with synergy and diversity, Semin. Immunol., 2009, 21, 130-138.

36 R. Akkerman, M. M. Faas and P. de Vos, Non-digestible carbohydrates in infant formula as substitution for human milk oligosaccharide functions: Effects on microbiota and gut maturation, Crit. Rev. Food Sci. Nutr., 2019, 59, 14861497.

37 K. P. Scott, J. C. Martin, C. Chassard, M. Clerget, J. Potrykus, G. Campbell, C.-D. Mayer, P. Young, G. Rucklidge, A. G. Ramsay and H. J. Flint, Substratedriven gene expression in Roseburia inulinivorans: Importance of inducible enzymes in the utilization of inulin and starch, Proc. Natl. Acad. Sci. U. S. A., 2011, 108, 4672-4679.

38 R. J. Palframan, G. R. Gibson and R. A. Rastall, Carbohydrate preferences of Bifidobacterium species isolated from the human gut, Curr. Issues Intest. Microbiol., 2003, 4, 71-75.

39 D. Vandeputte, G. Falony, S. Vieira-Silva, J. Wang, M. Sailer, S. Theis, K. Verbeke and J. Raes, Prebiotic inulin-type fructans induce specific changes in the human gut microbiota, Gut, 2017, 66, 1968-1974.

40 S. H. Kim, D. H. Lee and D. Meyer, Supplementation of baby formula with native inulin has a prebiotic effect in formula-fed babies, Asia Pac. J. Clin. Nutr., 2007, 16, 172177.

41 W. K. W. Yap, S. Mohamed, M. Husni Jamal, M. Diederick and Y. A. Manap, Changes in infants faecal characteristics and microbiota by inulin supplementation, J. Clin. Biochem. Nutr., 2008, 43, 159-166.

42 E. Elison, L. K. Vigsnaes, L. Rindom Krogsgaard, J. Rasmussen, N. Sørensen, B. McConnell, T. Hennet, M. O. Sommer and P. Bytzer, Oral supplementation of healthy adults with 2'-O-fucosyllactose and lacto-N-neotetraose is well tolerated and shifts the intestinal microbiota, Br. J. Nutr., 2016, 116, 1356-1368.

43 M. M. P. Oerlemans, R. Akkerman, M. Ferrari, M. T. C. Walvoort and P. de Vos, Benefits of bacteriaderived exopolysaccharides on gastrointestinal microbiota, immunity and health, J. Funct. Foods, 2021, 76, 104289.

44 J. H. C. Yiu, B. Dorweiler and C. W. Woo, Interaction between gut microbiota and toll-like receptor: from immunity to metabolism, J. Mol. Med., 2017, 95, 13-20.

45 N. Castro-Bravo, J. M. Wells, A. Margolles and P. RuasMadiedo, Interactions of surface exopolysaccharides from Bifidobacterium and Lactobacillus, within the intestinal environment, Front. Microbiol., 2018, 9, 2426.

46 L. Hussaarts, M. Yazdanbakhsh and B. Guigas, Priming dendritic cells for Th2 polarization: lessons learned from helminths and implications for metabolic disorders, Front. Immunol., 2014, 5, 499.

47 R. Divekar and H. Kita, Recent advances in epitheliumderived cytokines (IL-33, IL-25, and thymic stromal lymphopoietin) and allergic inflammation, Curr. Opin. Allergy Clin. Immunol., 2015, 15, 98-103.

48 I. Debock and V. Flamand, Unbalanced neonatal CD4+Tcell immunity, Front. Immunol., 2014, 5, 393. 